\title{
A New Transmitter-Receiver Architecture for Noncoherent Multirate OFFH-CDMA System With Fixed Optimal Detection Threshold
}

\author{
Elie Inaty, Member, IEEE, Hossam M. H. Shalaby, Senior Member, IEEE, and Paul Fortier, Senior Member, IEEE
}

\begin{abstract}
This paper analyzes a new transmitter-receiver architecture based on a modified version of unipolar-bipolar correlation proposed for a noncoherent multirate optical fast frequency hopping code-division multiple-access (OFFH-CDMA) system. The system assigns a frequency-shifted version (FSV) of the code used to transmit data bit "1" in order to transmit data bit " 0. " For the original system, we show that due to the nature of the multimedia network, the fluctuation in the multiple-access interference (MAI) average causes a threshold drift and thus an increase in the probability of error. This paper also provides a stochastic description of the MAI average amplitude fluctuation using a predefined multimedia probability density function. A system model is presented; in addition, the signal-to-interference ratio is derived. From the theoretical analysis and numerical results, it is shown that the proposed system has good performance without dynamic estimation of the detection threshold, and thus is independent of both the number of users and the distribution of those users in the offered multimedia classes.
\end{abstract}

Index Terms-Fiber Bragg grating, multimedia network, multirate, optical fast frequency hopping code-division multiple access (OFFH-CDMA), power control function.

\section{INTRODUCTION}

$\mathbf{L}$ ATELY, there is a growing interest in the development of broadband optical fiber communication networks using code-division multiple access (CDMA) for multimedia applications. Such networks must support heterogeneous traffic like high-speed and low-speed data, text, image, audio, and video, with varieties of quality of service (QoS) and traffic requirements [1]-[3].

An optical fast frequency hopping CDMA (OFFH-CDMA) system using fiber Bragg gratings and direct detection has been investigated in [3] and [4]. In this system, each user is assigned one sequence for transmission; the sequence is employed to transmit a " 1 " while nothing is transmitted for a " 0 ." Due to the unpredictable system activities in [3], the average of the multiple-access interference (MAI) will be unpredictable. Because the optimal threshold is a function of the average MAI, it will fluctuate depending on the system activities in terms of the number of users. This problem was previously considered in

Manuscript received May 31, 2001; revised July 30, 2002.

E. Inaty is with the Department of Electrical and Computer Engineering, University of Balamand, EL-Koura, Lebanon, (e-mail: elie.inaty@balamand.edu.lb).

H. M. H. Shalaby is with the Department of Electrical Engineering, University of Alexandria, 21544 Alexandria, Egypt (e-mail: shalaby@ieee.org).

P. Fortier is with the Department of Electrical and Computer Engineering, Laval University, G1K 7P4 QC, Canada (e-mail: fortier@gel.ulaval.ca).

Digital Object Identifier 10.1109/JLT.2002.806358

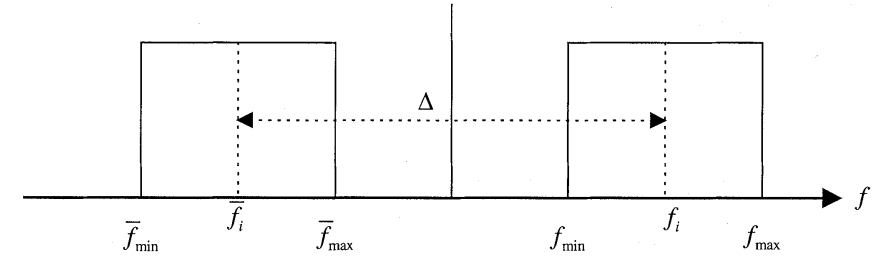

Fig. 1. Bandwidth of the proposed system.

[6] for direct-sequence CDMA (DS-CDMA). In multimedia applications, another problem contributes greatly to the dynamic variation of the optimal detection threshold, namely, users have the possibility of changing their traffic types dynamically, as proposed in [3]. We will show that the average MAI is a function not only of the active number of users but also of the processing gain (PG), the power-control function used, and the distribution of the active users between different multimedia classes.

To solve this problem, we proposed in this paper a new system architecture based on the one proposed in [3]. In this new architecture, each user is assigned two mutually orthogonal two-dimensional code sequences with equal PG. One is designated for "0" transmission, and its orthogonal version is used for transmitting data "1." In [5] and [6], equal-weight orthogonal (EWO) signaling was used. The orthogonal sequence was generated using a time-shifted reverse version of the original sequence. On the other hand, in our system, the orthogonality between the two versions is achieved by using a frequency-shifted version (FSV) of the original code in a way that no frequency overlap exists between the two codes, as shown in Fig. 1. This method allows an extra degree of flexibility especially in multirate systems where codes with different lengths are used and the time-shifted reverse version may lead to higher cross-correlation and out-of-phase autocorrelation [1].

The rest of this paper is organized as follows. In Section II, we present a model of the original system, where we show the dependence of the optimal detection threshold on the average MAI using the Bayes Risk criteria. In Section III, we present the modified system based on the FSV. In addition, we derive its signal-to-interference ratio (SIR) expression using the same technique adopted for the original system, but with a simple time-frequency transformation, and we show that its performance is independent of the average MAI. In Section IV, we analyze the random fluctuation of the average MAI in a multimedia network and derive a stochastic expression for a newly defined parameter called the threshold drift. Moreover, we derive the worst case multimedia 
distribution that causes the highest threshold drift, and equivalently the worst performance. An analytical performance comparison between the original and the newly proposed system is shown in Section V. In Section VI, a numerical simulation is presented. We conclude in Section VII.

\section{ORIGINAL SYSTEM}

\section{A. System Model}

Consider a fiber-optic CDMA communication network with transmitter/receiver pairs using OFFH-CDMA [3]. This system supports $K$ users, which share the same optical medium in a star architecture. Each of the $K$ users has the possibility of switching its traffic rate for any of $S$ possible values $R_{0}<R_{1}<$ $\cdots<R_{S-1}$ corresponding to $S$ different types of multimedia traffic or $S$ different classes. The corresponding PGs are given by $G_{0}>G_{1}>\cdots>G_{S-1}$, where $G_{i}=T_{i} / T_{c} . T_{i}$ and $T_{c}$ are the bit period and chip duration, respectively. The transmission power of a class-s, $\forall s \in\{0,1, \ldots, S-1\}$, is given by $P^{(s)}$. In addition, assume that the total number of active class- $s$ users is $K_{s}$ with $\sum_{s=0}^{S-1} K_{s}=K$.

\section{B. Average SIR}

The derivation to obtain the system SIR is similar to the analysis presented in [7], and thus it will not be fully presented here. Using the model presented in [7], the SIR experienced by an active user that has rate $R_{m}$ is

$$
\operatorname{SIR}_{m}=\frac{G_{m}^{2}}{\sum_{k=1}^{K_{m}-1} \sigma_{m, m}^{2}+\sum_{\substack{s=0 \\ s \neq m}}^{S-1} \sum_{k=0}^{K_{s}-1}\left(\frac{P^{(s)}}{P^{(m)}}\right) \sigma_{m, s}^{2}+\sigma_{n}^{2}}
$$

where $\sigma_{n}^{2}$ is the variance of a zero-mean additive white Gaussian noise (AWGN). On the other hand, $\sigma_{m, j}^{2} \forall j \in\{0,1, \ldots, S-$ $1\}$ represents the interference power caused by user $j$ from class $-j$ on the desired user $m$ from class- $m$ and is given by

$$
\sigma_{m, j}^{2}=\frac{1}{2 G_{j}}\left\{R_{k}\left[G_{m}, G_{j}\right]-\frac{1}{2 G_{j}} J_{k}^{2}\left[G_{m}, G_{j}\right]\right\} .
$$

In addition, $J_{k}\left[G_{m}, G_{j}\right]$ and $R_{k}\left[G_{m}, G_{j}\right]$ are the average and correlation parameters derived in [7] (for details, please refer to [7, (21) and (22), respectively]).

\section{From a Detection Theory Perspective}

Each source generates an output that in the simplest case is one of two choices, which are referred to as hypotheses labeled by $H_{0}$ and $H_{1}$. In optical CDMA systems, $H_{0}$ and $H_{1}$ correspond to the cases when "zero" and "one" are sent, respectively. To detect the desired signal in MAI plus noise, the two above-mentioned hypotheses can be defined as follows: $H_{0}-$ MAI plus noise is received and $H_{1}$ - signal $S_{D}$ plus $M A I$ plus noise is received. Thus, the decision at the $i$ th receiver, which is tuned to the $i$ th transmitter that transmits using rate $R_{s}$ and power $P^{(s)}$, is $Z_{i}$, which can be treated as a random variable whose statistical properties under both hypotheses are known. To make the detection approach clearer and to show the importance of the detection threshold in the original system, we assume that our detector follows the Bayesian hypothesis testing procedure [10].

Consider a system with the two hypotheses concerning a real observation $Z_{i}$ mentioned before

$$
\begin{aligned}
& H_{0}: Z_{i}=I_{m}+\mu_{0} \\
& H_{1}: Z_{i}=I_{m}+\mu_{1}
\end{aligned}
$$

where $I_{m}$ is the total MAI plus the background noise, which is modeled as a Gaussian random variable with mean $\mu_{\mathrm{MAI}}$ and variance $\sigma^{2}$, which are given by

$$
\begin{aligned}
\mu_{\mathrm{MAI}}= & \sqrt{P^{(m)}} \sum_{k=1}^{K_{m}-1} J_{k}\left[T_{m}, T_{m}\right] \\
& +\sum_{\substack{s=0 \\
s \neq m}}^{S-1} \sqrt{P^{(s)}} \sum_{k=0}^{K_{s}-1} J_{k}\left[T_{m}, T_{s}\right] \\
\sigma^{2}= & \sigma_{\mathrm{MAI}}^{2}+\sigma_{n}^{2} \\
= & P^{(m)} \sum_{k=1}^{K_{m}-1}\left(R_{k}\left[T_{m}, T_{m}\right]-J_{k}^{2}\left[T_{m}, T_{m}\right]\right) \\
& +\sum_{\substack{s=0 \\
s \neq m}}^{S-1} P^{(s)} \sum_{k=0}^{K_{s}-1}\left(R_{k}\left[T_{m}, T_{s}\right]-J_{k}^{2}\left[T_{m}, T_{s}\right]\right)+\sigma_{n}^{2} .
\end{aligned}
$$

The two parameters $\mu_{0}$ and $\mu_{1}$ are two fixed numbers given by $\mu_{0}=0$ and $\mu_{1}=S_{D}$. Note that the addition of $\mu_{0}$ or $\mu_{1}$ to $I_{m}$ changes only the mean value of the observation. In terms of distributions on the observation space, the hypothesis pair of (3) can be rewritten as

$$
\begin{aligned}
& H_{0}: Z_{i} \sim p_{0}\left(z_{i}\right)=N\left(\mu_{0}+\mu_{\mathrm{MAI}}, \sigma^{2}\right) \\
& H_{1}: Z_{i} \sim p_{1}\left(z_{i}\right)=N\left(\mu_{1}+\mu_{\mathrm{MAI}}, \sigma^{2}\right)
\end{aligned}
$$

where $N(a, b)$ denotes the normal distribution with mean $a$ and variance $b$. The likelihood ratio statistic [10] for (6) is

$$
L\left(z_{i}\right)=\frac{p_{1}\left(z_{i}\right)}{p_{0}\left(z_{i}\right)}=\exp \left\{\frac{S_{D}}{\sigma_{\mathrm{MAI}}^{2}}\left(z_{i}-\frac{2 \mu_{\mathrm{MAI}}+S_{D}}{2}\right)\right\} .
$$

As assumed in [7], the data are equiprobable. In addition, we assume uniform cost. Using those constraints, we can define the Bayes risk as the overall average cost incurred by a given decision rule $\pi$ as

$$
r(\pi)=\frac{1}{2}\left\{1+\int_{\Gamma_{1}} p_{0}\left(z_{i}\right) d z_{i}-\int_{\Gamma_{1}} p_{1}\left(z_{i}\right) d z_{i}\right\}
$$

where $\Gamma_{1}$ is the critical region, which is defined by

$$
\Gamma_{1}=\left\{z_{i} \in \Gamma \mid L\left(z_{i}\right) \geq \eta\right\}
$$

$\Gamma$ is the observation space and $\eta$ is equal to one given that we assume equiprobable data and uniform cost. Due to the fact that $L\left(z_{i}\right)$ is an increasing function of $z_{i}$, (9) can be written as

$$
\Gamma_{1}=\left\{z_{i} \in \Gamma \mid z_{i} \geq \eta_{\mathrm{opt}}\right\}
$$

where $\eta_{\mathrm{opt}}$ is the optimal detection threshold and can be easily shown to be

$$
\eta_{\mathrm{opt}}=\mu_{\mathrm{MAI}}+\frac{S_{D}}{2}
$$




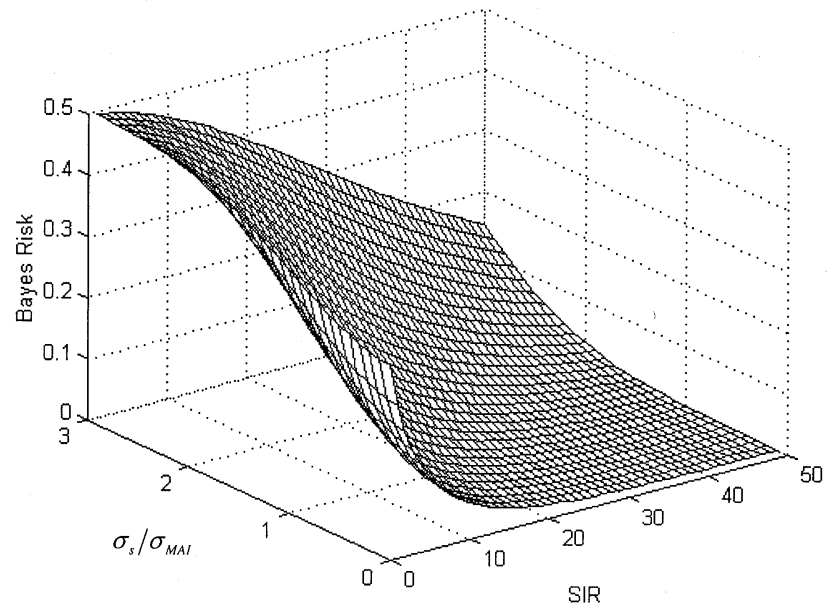

Fig. 2. Bayes risk for an active user versus the SIR and the ratio $\sigma_{s} / \sigma_{\mathrm{MAI}}$.

When the system activities change dynamically, the MAI average can be modeled as $m_{\mathrm{MAI}}=\mu_{\mathrm{MAI}}+\sigma_{s}$, where $\sigma_{s}$ is the threshold drift, which will be estimated in Section IV. Thus, the Bayes risk becomes

$$
\begin{aligned}
r(\pi)=\frac{1}{2}\left\{1+\Phi\left(-\frac{\sqrt{\mathrm{SIR}}}{2}\right.\right. & \left.-\frac{\sigma_{s}}{\sigma_{\mathrm{MAI}}}\right) \\
& \left.-\Phi\left(\frac{\sqrt{\mathrm{SIR}}}{2}-\frac{\sigma_{s}}{\sigma_{\mathrm{MAI}}}\right)\right\}
\end{aligned}
$$

where $\Phi$ denotes the cumulative probability distribution function of $N(0,1)$ random variable. Note that if the detector tracks the stochastic variation in the average MAI, $\sigma_{s}=0$ and the Bayes risk takes on its absolute minimum value, which is given by

$$
r_{\min }(\pi)=1-\Phi\left(\frac{\sqrt{\mathrm{SIR}}}{2}\right) .
$$

Fig. 2 shows the Bayes risk for any active user versus the SIR and the ratio $\sigma_{s} / \sigma_{\mathrm{MAI}}$. It is clear that when $\sigma_{s} / \sigma_{\mathrm{MAI}}$ becomes higher, the risk of an error becomes also higher. In addition, it is normal that when the SIR increases, the risk tends asymptotically to very small values.

\section{MODIFIED SYSTEM}

\section{A. System Model}

Consider the same fiber-optic CDMA communication network used in the previous section [3], [4] with a new transmitter/receiver pairs using OFFH-CDMA. Each user is assigned two mutually orthogonal sequences with equal weight generated from the same family of codes using an FSV as the orthogonal sequence. These orthogonal sequences are assigned to the transmission of " 0 " and " 1 ," respectively. In fact, the orthogonality between the sequences is achieved by choosing each of them from two different frequency bands so that no frequency hit may exist, although they are from the same family of codes. Fig. 3(a) shows the transmitter architecture using the FSV of the FFH code signaling. It is practically realized using two encoders, which represent a series of fiber Bragg gratings tuned to the desired user code $a_{k}^{(s)}(t, f)$ and its orthogonal version $\bar{a}_{k}^{(s)}(t, f)$. Notice that the overbar means the FSV. The data are fed into the encoders using two wide-band sources. Thus, the data bits are modulated either by the sequence $a_{k}^{(s)}(t, f)$ or its FSV $\bar{a}_{k}^{(s)}(t, f)$ depending on whether it is a " 1 " or " 0 ," respectively. The two codes are shown in Fig. 3(b). They are related by $a_{k}^{(s)}(t, f)=\bar{a}_{k}^{(s)}(t, f+\Delta)$, where $\Delta$ is the frequency shift. In addition, $\bar{f}_{\max }$ must be less than $f_{\min }$.

Fig. 3(c) shows the receiver of the modified system, which is practically realized by a $1: 2$ coupler and two matched filters. The lower branch filter is a correlator matched to $a_{k}^{(s)}(t, f)$, and the upper branch filter is matched to $\bar{a}_{k}^{(s)}(t, f)$. The outputs of the two correlators are subtracted via a balanced photodiode. The transmitted signal $S_{k}(t, f)$ can be expressed as

$$
S_{k}(t, f)=\sqrt{P^{(s)}} \bar{a}_{k}^{(s)}\left(t, f+\Delta b_{k}^{(s)}(t)\right)
$$

where $b_{k}^{(s)}(t)$ is the baseband unipolar signal of values $\{0,1\}$ for traffic type $s$. Notice that when the data bit is " 0 ," the transmitted signal is $\sqrt{P^{(s)}} \bar{a}_{k}^{(s)}(t, f)$. On the other hand, when the data bit is " 1 ," the transmitted signal is $\sqrt{P^{(s)}} \bar{a}_{k}^{(s)}(t, f+\Delta)=$ $\sqrt{P^{(s)}} a_{k}^{(s)}(t, f)$.

The received signal at each receiver can be written as

$$
\begin{aligned}
y(t, f) & =\sum_{k=0}^{K-1} S_{k}\left(t-\tau_{k}, f\right)+n(t) \\
& =\sum_{k=0}^{K-1} \sqrt{P^{(s)}} \bar{a}_{k}^{(s)}\left(t-\tau_{k}, f+\Delta b_{k}^{(s)}\left(t-\tau_{k}\right)\right)+n(t)
\end{aligned}
$$

where $\tau_{k}$ is the time delay associated with the $k$ th signal and $n(t)$ is AWGN with two-sided spectral density $N_{0} / 2$. The signal at the output of the adder is averaged over the bit duration $T_{m}$ by an integrate-and-dump low-pass filter. Therefore, the decision variable at the filter output is

$$
\begin{aligned}
Z_{0}^{(m)}=N+\frac{1}{(1+\alpha)} & \sum_{s=0}^{S-1} \sum_{k=0}^{K_{s}-1} \int_{o}^{T_{m}} S_{k}\left(t-\tau_{k}, f\right) \\
& \cdot\left[a_{0}^{(m)}(t, f)-\alpha \bar{a}_{0}^{(m)}(t, f)\right] d t
\end{aligned}
$$

where $N$ is a zero-mean AWGN with two-sided spectral density $N_{1} / 2$ that represents the thermal noise at the receiver with variance $\sigma_{n}^{2}=N_{1} T_{m} / 4$ and $T_{m}$ is the bit period corresponding to rate $R_{m}$.

To eliminate the frequency dimension of the signals, we use the Hamming correlation concept used in [7]. This enables us to write (16) as

$$
\begin{aligned}
Z_{0}^{(m)}= & N+\frac{1}{(1+\alpha)} \sum_{s=0}^{S-1} \sum_{k=0}^{K_{s}-1} \int_{o}^{T_{m}} \sqrt{P^{(s)}} h \\
& \cdot\left(\bar{a}_{k}^{(s)}\left(t-\tau_{k}\right)+\Delta b_{k}\left(t-\tau_{k}\right), a_{0}^{(m)}(t)\right) d t \\
& -\frac{\alpha}{(1+\alpha)} \sum_{s=0}^{S-1} \sum_{k=0}^{K_{s}-1} \int_{0}^{T_{m}} \sqrt{P^{(s)}} h \\
& \cdot\left(\bar{a}_{k}^{(s)}\left(t-\tau_{k}\right)+\Delta b_{k}\left(t-\tau_{k}\right), \bar{a}_{0}^{(m)}(t)\right) d t .
\end{aligned}
$$




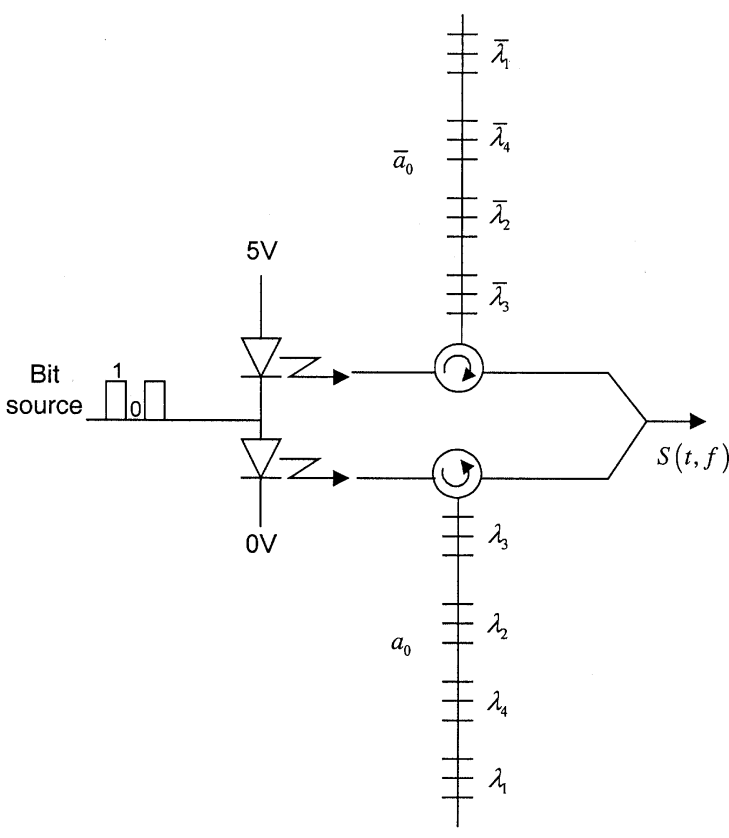

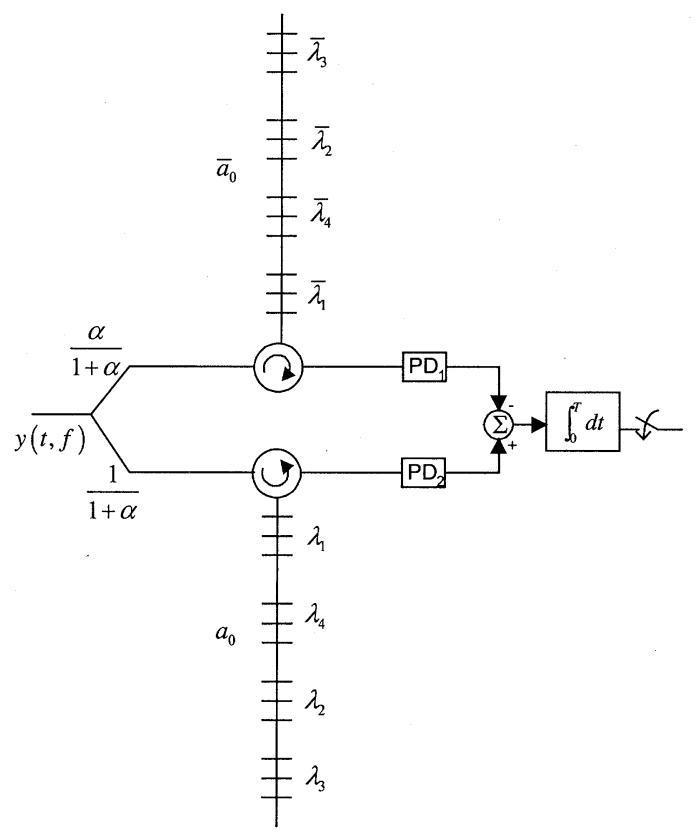

c)

Fig. 3. (a) Modified OFFH-CDMA transmitter, (b) its corresponding 2D-code structure, and (c) modified OFFH-CDMA receiver.

The sequences $a_{k}^{(s)}(t), \bar{a}_{k}^{(s)}(t), a_{0}^{(m)}(t)$, and $\bar{a}_{0}^{(m)}(t)$ are real numbers representing the hopping frequencies along with their orthogonal versions used at time $t$ for the $k$ th interferer and the desired user, respectively.

\section{B. Average SIR}

Using this modified model, the desired signal component, assuming perfect synchronization between the receiver and the desired transmitter and balanced coupler $(\alpha=1)$, is $S_{D}=$ $\pm \sqrt{P^{(m)}} T_{m} / 2$ for $b_{0}^{(m)}=1$ and $b_{0}^{(m)}=0$, respectively. The MAI from user $k$ that transmits data with rate $R_{s}$ is

$$
\begin{gathered}
I_{k}=\frac{\sqrt{P^{(s)}}}{(1+\alpha)} \int_{0}^{T_{m}} h\left(\bar{a}_{k}^{(s)}\left(t-\tau_{k}\right)+\Delta b_{k}\left(t-\tau_{k}\right), a_{0}^{(m)}(t)\right) d t \\
-\frac{\alpha \sqrt{P^{(s)}}}{(1+\alpha)} \int_{0}^{T_{m}} h\left(\bar{a}_{k}^{(s)}\left(t-\tau_{k}\right)+\Delta b_{k}\left(t-\tau_{k}\right)\right. \\
\left.\bar{a}_{0}^{(m)}(t)\right) d t
\end{gathered}
$$

$I_{k}$ is assumed to be an independent random variable. Its variance can be written as $\sigma_{I_{k}}^{2}=E\left(I_{k}^{2}\right)-E^{2}\left(I_{k}\right)$, where $E($.$) is the$ expectation operator. We assume that $\tau_{k}$ and $b_{k}^{(s)}(t)$, for $1 \leq$ $k \leq K$, are mutually independent. $\tau_{k}$ is uniformly distributed over the range $0 \leq \tau_{k} \leq T_{s}$ and $\operatorname{Pr}\left[b_{k}^{(s)}=1\right]=\operatorname{Pr}\left[b_{k}^{(s)}=0\right]=$ $1 / 2$. Thus, averaging over $\tau_{k}$ and $b_{k}^{(s)}$, we obtain

$$
\begin{aligned}
& E\left(I_{k}\right)=\sqrt{P^{(s)}} J_{k}\left[T_{m}, T_{s}\right] \\
& E\left(I_{k}^{2}\right)=P^{(s)} R_{k}\left[T_{m}, T_{s}\right]
\end{aligned}
$$

where $J_{k}\left[T_{m}, T_{s}\right]$ and $R_{k}\left[T_{m}, T_{s}\right]$ are the average and correlation parameters. They must be derived for two different cases: 1) $T_{m} \leq T_{s}$ and 2) $T_{m}>T_{s}$.
1) $T_{m} \leq T_{s}$ : Averaging over all possible values of $b_{k,-1}^{(s)}$ and $b_{k, 0}^{(s)}$, we will obtain

$$
\begin{aligned}
J_{k}\left[T_{m}, T_{s}\right]= & \frac{(1-\alpha)}{(1+\alpha)} \frac{1}{2 T_{s}} \int_{0}^{T_{s}} H_{k, 0}\left(0, T_{m}\right) d \tau_{k} \\
R_{k}\left[T_{m}, T_{s}\right]= & \frac{\left(1+\alpha^{2}\right)}{(1+\alpha)^{2}} \frac{1}{2 T_{s}} \int_{0}^{T_{m}} H_{k, 0}^{2}\left(0, \tau_{k}\right) \\
& +H_{k 0}^{2}\left(\tau_{k}, T_{m}\right) d \tau_{k} \\
& +\frac{\left(1+\alpha^{2}\right)}{(1+\alpha)^{2}} \frac{1}{2 T_{s}} \cdot \int_{T_{m}}^{T_{s}} H_{k, 0}^{2}\left(0, T_{m}\right) d \tau_{k} \\
& +\frac{(1-\alpha)}{(1+\alpha)^{2}} \frac{1}{2 T_{s}} \\
& \cdot \int_{0}^{T_{m}} H_{k, 0}\left(0, \tau_{k}\right) H_{k, 0}\left(\tau_{k}, T_{m}\right) d \tau_{k}
\end{aligned}
$$

where $H_{k, 0}\left(0, \tau_{k}\right), H_{k, 0}\left(\tau_{k}, T_{m}\right)$, and $H_{k, 0}\left(0, T_{m}\right)$ are given by

$$
H_{k, 0}\left(\tau_{i}, \tau_{j}\right)=\int_{\tau_{i}}^{\tau_{j}} h\left(a_{k}\left(t-\tau_{k}\right), a_{0}(t)\right) d t .
$$

2) $T_{m}>T_{s}$ : By taking $L=\left\lfloor\left(T_{m}-\tau_{k}\right) / T_{s}\right\rfloor$ and averaging over all possible values of the overlapping bits $b_{k,-1}^{(s)}, \ldots, b_{k, L}^{(s)}$, $R_{k}\left[T_{m}, T_{s}\right]$ and $J_{k}\left[T_{m}, T_{s}\right]$ can now be written as

$$
J_{k}\left[T_{m}, T_{s}\right]=\frac{(1-\alpha)}{(1+\alpha)} \frac{1}{2 T_{s}} \int_{0}^{T_{s}} H_{k, 0}\left(0, T_{m}\right) d \tau_{k}
$$

and (22), which can be found at the bottom of the next page.

By considering a balanced coupler $(\alpha=1)$ and using the discrete partial-period aperiodic Hamming-correlation func- 
tions defined in [7], $\mathrm{AH}_{1}, \mathrm{AH}_{2}, \mathrm{AH}_{3}$, and $\mathrm{AH}_{4}$ [7, (16)-(20)], $J_{k}\left[T_{m}, T_{s}\right]$ becomes equal to zero and $R_{k}\left[T_{m}, T_{s}\right]$ will be

$$
R_{k}\left[T_{m}, T_{s}\right]= \begin{cases}\frac{T_{c}^{3}}{4 T_{s}}\left\{\sum_{q=0}^{G_{m}-1}\left[\mathrm{AH}_{1}^{2}(q)+\mathrm{AH}_{2}^{2}\left(q, G_{m}\right)\right]\right. \\ \left.+\sum_{q=G_{m}}^{G_{s}-1} \mathrm{AH}_{3}^{2}\left(q, G_{m}\right)\right\}, & T_{m} \leq T_{s} \\ \frac{T_{c}^{3}}{4 T_{s}} \sum_{q=0}^{G_{s}-1}\left[\mathrm{AH}_{1}^{2}(q)+\mathrm{AH}_{2}^{2}\left(q+L G_{s}, G_{m}\right)\right. \\ \left.+\sum_{y=1}^{L} \mathrm{AH}_{4}^{2}\left(q, y, G_{s}\right)\right], & T_{m}>T_{s} .\end{cases}
$$

Therefore, the mean of the MAI vanishes to zero independently of the active number of users or the classes chosen by those users. In addition, the cross terms in (22) are forced to be equal to zero. Thus, the above result is consistent with the result obtained in [8] for coherent multirate CDMA.

If we define $R_{k}\left[G_{m}, G_{s}\right]=R_{k}\left[T_{m}, T_{s}\right] /\left(T_{c}^{2} / 4 G_{s}\right)$, the SIR experienced by an active user that has rate $R_{m}$ and, using the modified system, is the same as that defined in (1), but the variance $\sigma_{m, j}^{2} \forall j \in\{0,1, \ldots, S-1\}$ is now given by

$$
\sigma_{m, j}^{2}=\frac{1}{2 G_{j}} R_{k}\left[G_{m}, G_{j}\right]
$$

\section{Stochastic Amplitude Fluctuation of the AVERAGE MAI}

\section{A. Threshold Line Width}

This section provides a stochastic description of the MAI amplitude fluctuation using a predefined multimedia probability density function (pdf). Because every user may choose his class and modify it dynamically, we assume that the class- $s$ can be considered as a discrete random variable with a certain probability mass function (pmf) given by the probability $P_{s}=\operatorname{Pr}$ (user $k$ chooses the class-s) with $\sum_{s=0}^{S-1} P_{s}=1$.

Proposition 1: Given that the sequences used for each class respect the one-coincidence criteria with nonrepeating frequencies, the expected value of the average MAI generated from the $k$ th user that transmits using the class- $s$ is given by

$$
\mu_{I_{k}}(s)=\frac{T_{c}}{2} \sqrt{P^{(s)}}\left(\frac{G_{m}}{F}\right)
$$

where $F$ is the total number of available frequencies.

Proof: Given that the number of coincidences between the desired and interferer sequences is optimized to a maximum of one, it is clear that the probability of hit in the first chip position is

$$
P\left(H_{0}\right)=\frac{1}{F} .
$$

Given $P\left(H_{0}\right)$, the probability of hit in the second chip position can be written as

$$
\begin{aligned}
P\left(H_{1}\right) & =P\left(H_{1} / H_{0}\right) P\left(H_{0}\right)+P\left(H_{1} / \bar{H}_{0}\right) P\left(\bar{H}_{0}\right) \\
& =P\left(H_{1} / \bar{H}_{0}\right) P\left(\bar{H}_{0}\right) .
\end{aligned}
$$

In addition, because we have nonrepeating frequencies in the same sequence, we can write $P\left(H_{1} / \bar{H}_{0}\right)=1 /(F-1)$. Thus, $P\left(H_{1}\right)$ can be written as

$$
P\left(H_{1}\right)=\frac{1}{(F-1)} \frac{(F-1)}{F}=\frac{1}{F} .
$$

By induction, we can show that the probability of hit at any chip position $i$ will be

$$
P\left(H_{i}\right)=\frac{1}{F} .
$$

$$
\begin{aligned}
& R_{k}\left[T_{m}, T_{s}\right]=\frac{\left(1+\alpha^{2}\right)}{(1+\alpha)^{2}} \frac{1}{2 T_{s}} \int_{0}^{T_{s}}\left[H_{k, 0}^{2}\left(0, \tau_{k}\right)+H_{k, 0}^{2}\left(\tau_{k}+L T_{s}, T_{m}\right) \sum_{y=1}^{L} H_{k, 0}^{2}\left(\tau_{k}+(y-1) T_{s}, \tau_{k}+y T_{s}\right)\right] d \tau_{k} \\
& +\frac{(1-\alpha)}{(1+\alpha)^{2}} \frac{1}{2 T_{s}} \int_{0}^{T_{s}}\left[H_{k, 0}\left(0, \tau_{k}\right) H_{k, 0}\left(\tau_{k}+L T_{s}, T_{m}\right)\right. \\
& +\left(H_{k, 0}\left(0, \tau_{k}\right)+H_{k, 0}\left(\tau_{k}+L T_{s}, T_{m}\right)\right) H_{k, 0}\left(\tau_{k}, L T_{s}\right) \\
& +\frac{1}{2} \sum_{x=1}^{L} \sum_{\substack{y=1 \\
y \neq x}}^{L} H_{k, 0}\left(\tau_{k}+(y-1) T_{s}, \tau_{k}+y T_{s}\right) \\
& \left.\cdot H_{k, 0}\left(\tau_{k}+(x-1) T_{s}, \tau_{k}+x T_{s}\right)\right] d \tau_{k}
\end{aligned}
$$


Given (26) and assuming that the sequences assigned to different users are mutually independent, we can calculate the expected values of $\mathrm{AH}_{3}\left(q, G_{m}\right)$ as shown below

$$
\begin{aligned}
\overline{\mathrm{AH}_{3}}\left(q, G_{m}\right) & =\sum_{j=0}^{G_{m}-1} E\left(h\left(a_{j-q}^{k}, a_{j}^{0}\right)\right) \\
& =\sum_{j=0}^{G_{m}-1} \frac{1}{F}=\frac{G_{m}}{F} .
\end{aligned}
$$

Using this result, $J_{k}\left[G_{m}, G_{s}\right]$ can be approximated by its expected value. We obtain

$$
\bar{J}\left[G_{m}, G_{s}\right]=\frac{G_{m} G_{s}}{F} .
$$

The average of the MAI generated from the $k$ th user that transmits using class-s is given by $\mu_{I_{k}}(s)$

$$
\mu_{I_{k}}(s)=\frac{T_{c}}{2} \frac{\sqrt{P^{(s)}}}{G_{s}} \bar{J}\left[G_{m}, G_{s}\right] .
$$

Substituting (28) into (29), we obtain (25).

Due to the fact that we have considered the class-s as being a random variable, any function of the class- $s$ is also considered as a random variable. Thus, $\mu_{I_{k}}(s)$ is a random variable with mean $m$ and variance $\Delta^{2}$, which can be written as

$$
\begin{aligned}
m= & E_{s}\left(\mu_{I_{k}}(s)\right)=\frac{T_{c}}{2}\left(\frac{G_{m}}{F}\right)\left(\sum_{s=0}^{S-1} P_{s} \sqrt{P^{(s)}}\right) \\
\Delta^{2}= & E_{s}\left(\mu_{I_{k}}^{2}(s)\right)-m^{2} \\
= & \left(\frac{T_{c}}{2}\right)^{2}\left(\frac{G_{m}}{F}\right)^{2} \\
& \cdot\left\{\sum_{s=0}^{S-1} P_{s} P^{(s)}-\sum_{s=0}^{S-1} \sum_{t=0}^{S-1} P_{s} P_{t} \sqrt{P^{(s)} P^{(t)}}\right\} .
\end{aligned}
$$

Since the users are assumed independent and may choose their classes also independently from each other, the mean $M_{s}$ and the variance $\sigma_{s}^{2}$ of the total MAI average are the sum of the means and variances from all active users, respectively, and they are given by

$$
\begin{aligned}
M_{s} & =(K-1) m \\
\sigma_{s}^{2} & =(K-1) \Delta^{2} .
\end{aligned}
$$

In this paper, the MAI average fluctuation is regarded as the square root of the variance or the standard deviation from the mean $\sigma_{s}$. The variable $\sigma_{s}$ is a measure of the amount of threshold drift, and we call it the threshold line width. The optimum threshold level given in (11) contains two terms. The first term is the desired signal and is supposed to be known from the destination. The second term is the average MAI, and the fluctuation of this term causes the amplitude fluctuation in the detection threshold. Thus, it induces a drift from the optimal threshold. Therefore, we can model the detection threshold at the receiver as follows:

$$
\eta=\frac{S_{D}}{2}+\mu_{\mathrm{MAI}} \pm \sigma_{s} .
$$

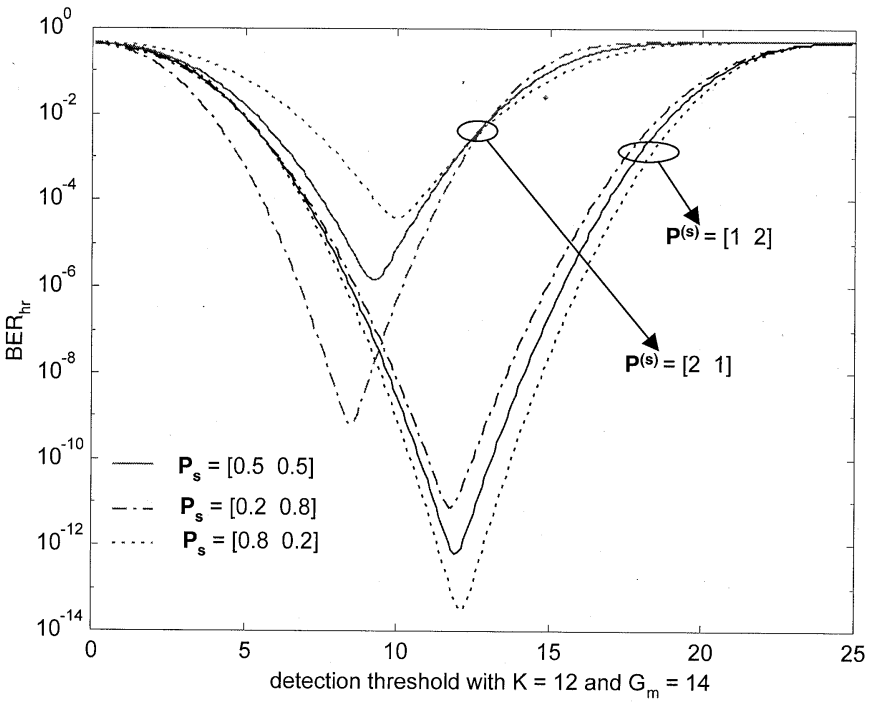

Fig. 4. The class- $h r$ BER versus the detection threshold for $K=12$.

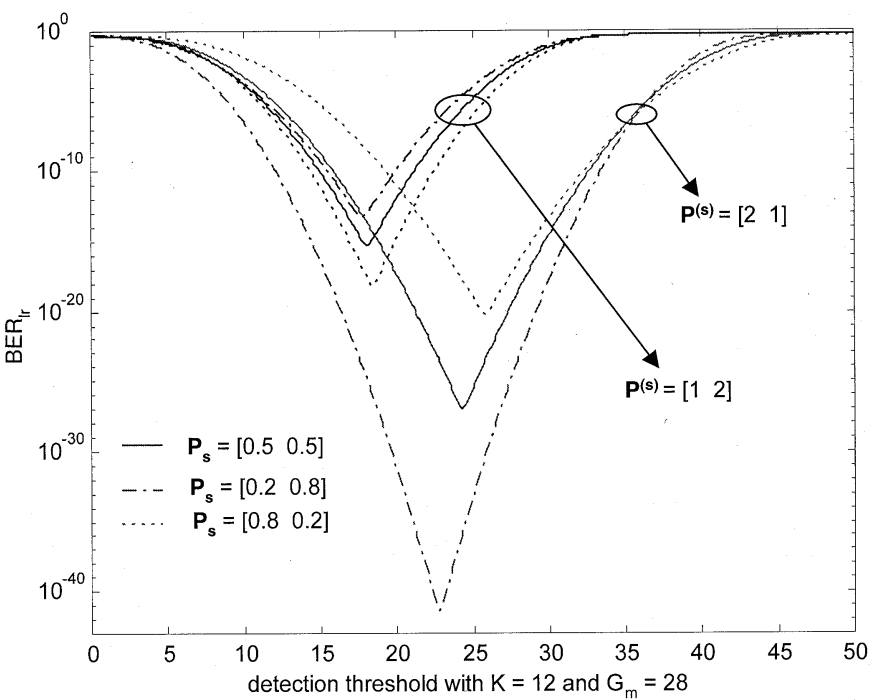

Fig. 5. The class-lr BER versus the detection threshold for $K=12$.

From (33), we can notice that the threshold drift is greatly dependent on the total number of users, the multimedia distribution, and the applied power-control function.

In Figs. 4 and 5, we plot the bit error rate (BER) versus the detection threshold for the case of a two-class system, namely, class- $h r$ and class-lr, respectively, and using the extended hyperbolic congruential (EHC) family of codes [9] with $F=41$ available frequencies. One can notice clearly the dependence of the BER and the $\eta_{\mathrm{opt}}$ on the PG of the desired user, the transmission power, and the multimedia pmf adopted in the network, assuming a fixed number of users $(K=12)$. In these figures, $\mathbf{P}_{s}=\left[\begin{array}{ll}P_{l r} & P_{h r}\end{array}\right]$ and $\mathbf{P}^{(s)}=\left[\begin{array}{ll}P^{(l r)} & P^{(h r)}\end{array}\right]$ represent vectors containing the pmf and the transmission power of each class, respectively. We can observe the variation of $\eta_{\mathrm{opt}}$ when we vary the transmission power of the two classes. Therefore, in a multirate system, the optimal threshold will fluctuate with the applied optimal power-control function [3]. 


\section{B. Worst Case Multimedia Distribution}

For a given power-control function, the multimedia distribution can be considered as a noise distribution that introduces threshold drift, which in turn causes higher probability of error. It will be interesting to find the worst case performance achievable by any multimedia distribution as a function of the applied power-control function. This distribution is called the worst case distribution.

In this section, the functional that quantifies the threshold drift is

$$
\Delta^{2}\left(\mathbf{P}_{\mathbf{S}}\right)=\rho\left(\mathbf{P}^{\mathbf{S}} \mathbf{P}_{\mathbf{S}}+\mathbf{P}_{\mathbf{S}}^{\mathbf{T}} \mathbf{A} \mathbf{P}_{\mathbf{S}}\right)
$$

where $\mathbf{P}_{\mathbf{S}}=\left[P_{0} P_{1} \cdot \cdot P_{S-1}\right]^{T}$ and $\mathbf{P}^{\mathbf{S}}=\left[P^{(0)} P^{(1)} \cdot\right.$ $\left.P^{(S-1)}\right]$. Notice that $\rho$ is a constant that depends on the desired user PG and the number of available frequencies. It is given by

$$
\rho=\left(\frac{T_{c}}{2}\right)^{2}\left(\frac{G_{m}}{F}\right)^{2}
$$

In addition, the matrix $\mathbf{A}$ is symmetric and is given by

$$
\begin{aligned}
& \mathbf{A}= \\
& {\left[\begin{array}{cccc}
-P^{(0)} & -\sqrt{P^{(0)} P^{(1)}} & \cdot & -\sqrt{P^{(0)} P^{(S-1)}} \\
-\sqrt{P^{(0) P^{(1)}}} & -P^{(1)} & \cdot & -\sqrt{P^{(S-1) P^{(1)}}} \\
\cdot & \cdot & \cdot & \cdot \\
\cdot & \cdot & \cdot & \cdot \\
-\sqrt{P^{(0) P^{(S-1)}}} & -\sqrt{P^{(1) P^{(S-1)}}} & \cdot & -P^{(S-1)}
\end{array}\right] .}
\end{aligned}
$$

The optimization problem is a nonlinear problem with linear constraints and can be stated as follows:

$$
\mathbf{P}_{\mathbf{S}}^{*}=\underset{P_{0}, P_{1}, \ldots, P_{S-1}}{\arg \max } \Delta^{2}\left(\mathbf{P}_{\mathbf{S}}\right)
$$

subject to

$$
\sum_{s=0}^{S-1} P_{s}=1 \quad P_{s} \geq 0 \quad \forall s \in\{0,1, \ldots, S-1\} .
$$

The constraint set in (37) can be considered as convex. Then it is sufficient that the objective function in (35) be a concave function in order that the global maximum will satisfy the Kuhn-Tucker (KT) conditions [11]. In fact, $\Delta^{2}\left(\mathbf{P}_{\mathbf{S}}\right)$ has a well-known quadratic form. From quadratic programming theory [11], we can say that $\Delta^{2}\left(\mathbf{P}_{\mathbf{S}}\right)$ is a concave function if $\mathbf{A}$ is a negative definite matrix, which means that this matrix is defined such that $\mathbf{P}_{\mathbf{S}}^{\mathbf{T}} \mathbf{A} \mathbf{P}_{\mathbf{S}}<0$ for all $\mathbf{P}_{\mathbf{S}}$ except $\mathbf{P}_{\mathbf{S}}=0$; this is the case in (35). For this reason, KT constraint qualification is automatically satisfied.

Proposition 2: Suppose that the power vector $\mathbf{P}^{\mathbf{S}}$ is defined such that $P^{(t)} \neq P^{(k)}$ for $t \neq k$. The least favorable multimedia distribution is given by a mixture of two probability masses at $s=i$ and $s=j$ with equal probabilities $P_{i}=P_{j}=1 / 2$, whose respective locations depend on the power vector $\mathbf{P}^{\mathrm{S}}$ such that

$$
\begin{aligned}
& P^{(i)}=\max \left\{P^{(0)}, P^{(1)}, \ldots, P^{(S-1)}\right\} \\
& P^{(j)}=\min \left\{P^{(0)}, P^{(1)}, \ldots, P^{(S-1)}\right\} .
\end{aligned}
$$

In addition, the maximum value of $\Delta^{2}\left(\mathbf{P}_{\mathbf{S}}\right)$ is given by

$$
\Delta_{\max }^{2}=\rho \frac{\left(\sqrt{P^{(i)}}-\sqrt{P^{(j)}}\right)^{2}}{4} .
$$

Proof: It is clear that $\mathbf{P}^{\mathbf{S}} \mathbf{P}_{\mathbf{S}}+\mathbf{P}_{\mathbf{S}}^{\mathbf{T}} \mathbf{A} \mathbf{P}_{\mathbf{S}}$ represents the variance of the square root of the power $\sqrt{P^{(s)}}$ with respect to the distribution $P_{s}$. Lemma 1 in the Appendix shows that the maximum variance of a finite discrete random variable is achieved by putting two masses of probability $1 / 2$ at the two extremes. This enables us to say that the maximum of the variance of the power is achieved when putting two masses of values $1 / 2$ at the extremes, thus at $P^{(i)}$ and $P^{(j)}$, respectively. Therefore, $P_{s}=1 / 2$ for $s \in\{i, j\}$ and zero elsewhere. For this reason, $\Delta_{\max }^{2}$ is given by (38).

\section{PERFORMANCE COMPARISON}

Basically, the only difference between the SIR expressions of the two systems is the value of the variances $\sigma_{m, j}^{2}$ shown in (2) and (24) for the original and the modified system, respectively. The system that processes the smallest variance will have the largest SIR and equivalently the better performance. Thus, by knowing the sign of the difference between the variances of the two systems, $d=\sigma_{m, j_{-} \mathrm{O}}^{2}-\sigma_{m, j_{-} \mathrm{M}}^{2}$ (the letters $\mathrm{O}$ and $\mathrm{M}$ stands for original and modified, respectively), we can figure out which of the two systems has the best performance. The parameter $d$ can be written as

$$
d\left(G_{m}, G_{s}\right)=\left\{\begin{array}{l}
\frac{1}{2 G_{s}} \sum_{q=0}^{G_{m}-1} \mathrm{AH}_{1}(q) \mathrm{AH}_{2}\left(q, G_{m}\right) \\
-\left[\frac{1}{2 G_{s}} \sum_{q=0}^{G_{s}-1} \mathrm{AH}_{3}\left(q, G_{m}\right)\right]^{2}, T_{m} \leq T_{s} \\
+\frac{1}{2} \sum_{x=1}^{L} \sum_{y=1}^{L} \mathrm{AH}_{4}\left(q, y, G_{s}\right) \mathrm{AH}_{4}\left(q, x, G_{s}\right) \\
+\left(\sum _ { y = 0 } ^ { G _ { s } - 1 } \left[\mathrm{AH}_{1}(q)+\mathrm{AH}_{2}\left(q+L \mathrm{AH}_{2}\left(q+L G_{s}, G_{m}\right)\right.\right.\right. \\
\left.\cdot \mathrm{AH}_{5}\left(q, L, G_{s}\right)\right) \\
-\left[\frac{1}{2 G_{s}} \sum_{q=0}^{G_{s}-1} \mathrm{AH}_{3}\left(q, G_{m}\right)\right]^{2}, T_{m}>T_{s} .
\end{array}\right.
$$

Proposition 3: For one-coincidence sequences where the maximum cross-correlation and out-of-phase autocorrelation are upper bounded by one, $\mathrm{SIR}_{\mathrm{O}} \geq \mathrm{SIR}_{\mathrm{M}}$ and, equivalently, BER $_{\mathrm{O}} \leq \mathrm{BER}_{\mathrm{M}}$ for $T_{m} \leq T_{s}$ and $T_{m}>T_{s}$ assuming perfect threshold estimation. 
Proof: Given that the number of coincidences between the desired and interferer sequences is optimized to a maximum of one, it is clear that at maximum, one of the aperiodic correlation functions in (39) is equal to one and the others are equal to zero for a given value of the delay $q$. Thus, we can easily say that the cross terms are always equal to zero. Therefore, (39) is simplified to

$$
\begin{aligned}
d\left(G_{m}, G_{s}\right) & =-\left[\frac{1}{2 G_{s}} \sum_{q=0}^{G_{s}-1} \mathrm{AH}_{3}\left(q, G_{m}\right)\right]^{2} \forall T_{m}, T_{s} \\
& \leq 0 .
\end{aligned}
$$

This implies that $\sigma_{m, j_{-} \mathrm{O}}^{2} \leq \sigma_{m, j_{-} \mathrm{M}}^{2}$ and, equivalently, $\operatorname{SIR}_{\mathrm{O}} \geq$ $\mathrm{SIR}_{\mathrm{M}}$.

Proposition 4: Assuming perfect threshold estimation and constant probability of hit $p$ for every chip position, the average value of the difference parameter $\bar{d}\left(G_{m}, G_{s}\right)$ is always negative; thus $\overline{\sigma_{\mathrm{O}}^{2}} \leq \overline{\sigma_{\mathrm{M}}^{2}}$ for $T_{m} \leq T_{s}$ and $T_{m}>T_{s}$. In addition, $\bar{d}\left(G_{m}, G_{s}\right)$ is a decreasing function of $p$.

Proof: Assume that the sequences exhibit sufficient randomness to consider that the probability of hit is constant and is equal to $p$. Using this hit probability, we can compute the average value of the partial correlation functions.

1) $T_{m} \leq T_{s}$ : The average value of the difference parameter can be written as

$$
\bar{d}\left(G_{m}, G_{s}\right)=\frac{p G_{m}}{4 G_{s}}\left[p G_{m}\left(\frac{G_{m}}{3}-G_{s}\right)-\left(1-\frac{2 p}{3}\right)\right] .
$$

Given that $G_{s} \geq G_{m}$, then $\left(\left(G_{m} / 3\right)-G_{s}\right)<0$. In addition, we know that $(1-(2 p / 3))>0$, which makes $\bar{d}\left(G_{m}, G_{s}\right)<0$ independently of the hit probability $p$, and thus, $\overline{\sigma_{\mathrm{O}}^{2}} \leq \overline{\sigma_{\mathrm{M}}^{2}}$. The derivative of $\bar{d}\left(G_{m}, G_{s}\right)$ with respect of $p$ is

$$
\frac{\partial \bar{d}\left(G_{m}, G_{s}\right)}{\partial p}=\frac{G_{m}}{4 G_{s}} 2 G_{m}\left(\frac{G_{m}}{3}-G_{s}\right) p-\left(1-\frac{4 p}{3}\right) \leq 0
$$

which proves that $\bar{d}\left(G_{m}, G_{s}\right)$ is a decreasing function of $p$.

2) $T_{m}>T_{s}$ : In this case, we consider that the sequence of the desired user is longer than that of the interferer

$$
\bar{d}\left(G_{m}, G_{s}\right)=-\frac{p}{12 G_{s}}\left[\left(3 G_{m}-G_{s}\right)\left(G_{s}^{2}-1\right) p+3 G_{m}\right] .
$$

Given that $G_{m}>G_{s}$ and $G_{s} \geq 1$, this will obviously make $\bar{d}\left(G_{m}, G_{s}\right)<0$ also independently of $p$. Then,

$$
\begin{aligned}
& \frac{\partial \bar{d}\left(G_{m}, G_{s}\right)}{\partial p} \\
& \quad=-\frac{1}{12 G_{s}}\left[2\left(3 G_{m}-G_{s}\right)\left(G_{s}^{2}-1\right) p+3 G_{m}\right] \leq 0
\end{aligned}
$$

which makes $\bar{d}$ a decreasing function of $p$. Hence the proof is completed.

The implication of Proposition 3 is straightforward in the sense that we are practically sure that whenever the one coincidence criteria is respected in the multirate system, the original

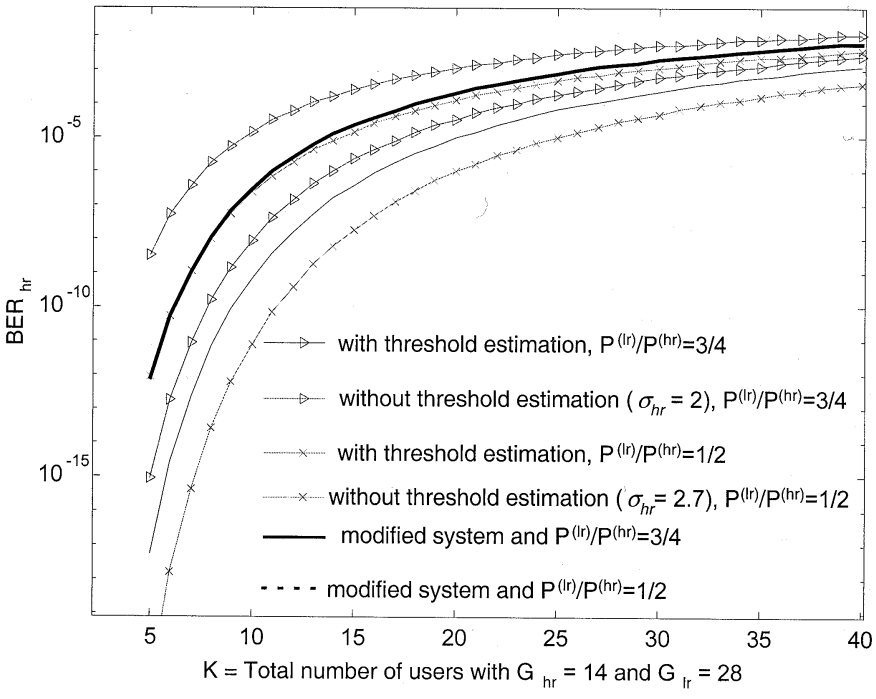

Fig. 6. The class-hr BER versus the total number of users, for the original and the modified systems.

system always outperforms the modified one, assuming optimal threshold estimation. Although Proposition 4 considers constant probability of hit at every chip position, it gives an important insight into the comparison problem by showing that on the average, the original system has better performance (assuming perfect threshold estimation) than the modified one independent from the hit probability. After all, the hit probability is related to the number of hits. If $p$ is high, the number hits is high too. This means that when the number of hits is high, the average performance of the original system will improve with respect to that of the modified one.

Although the BER of the modified system using FSV seems to increase with respect to the original system as the number of hits increases, we can notice that the threshold drift increases also as the $p$ increases. Therefore, if we consider a simple receiver that does not track the optimal threshold variations, the overall performance of the modified system will always outperform that of the original one.

\section{NUMERICAL RESULTS AND DISCUSSIONS}

Throughout this section, it must be noted that the same family of codes used previously is employed. Moreover, the powercontrol algorithm and the definition of throughput are used according to [3]. Briefly, the power-control algorithm is the algorithm that provides the optimal transmission power level for each class of users, which maximizes the system throughput. The throughput function was defined as the weighted sum of the number of users in each class. The QoS guarantee for each class is taken to be a lower bound on the SIR.

Figs. 6 and 7 show the BER performance comparison, between the two systems, for class- $h r$ and class-lr users, respectively, and using a multimedia pdf of $P_{h r}=P_{l r}=0.5$ while varying the power ratio $P^{(l r)} / P^{(h r)}$ to take values $3 / 4$ and $1 / 2$. It is clear that in both cases, the BER of the original system is better than that of the modified one when the detector tracks the optimal threshold. Note that when the number of users becomes high, the two BERs become closer. 


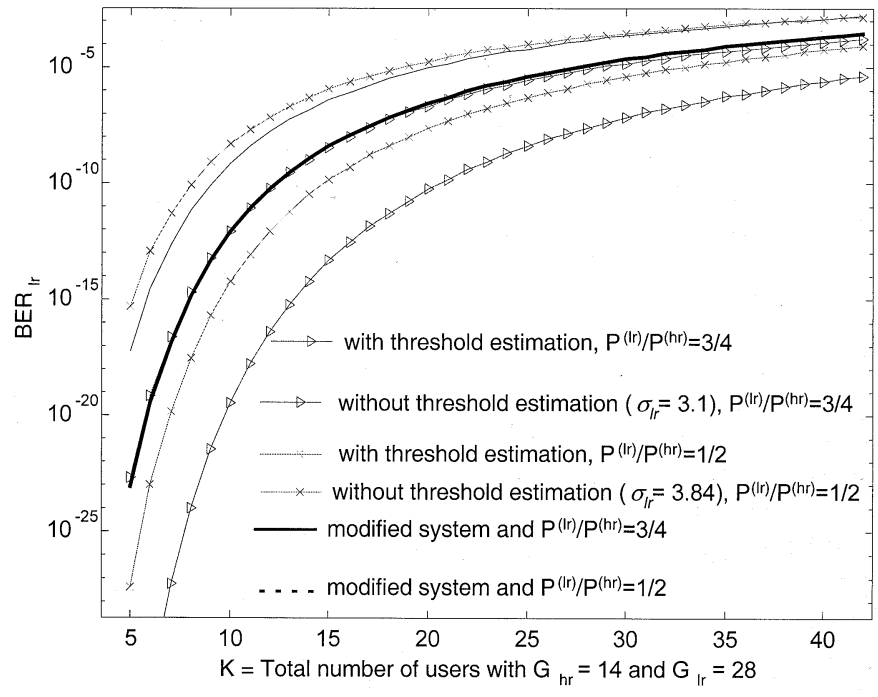

Fig. 7. The class-lr BER versus the total number of users for the original and the modified systems.

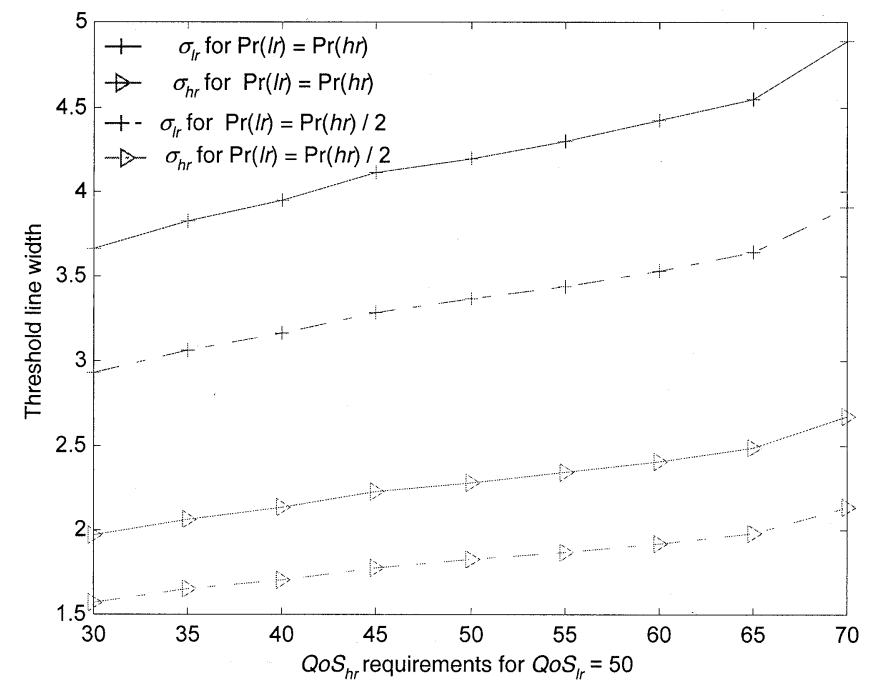

Fig. 8. Threshold line width versus the class-hr QoS requirement for fixed class-lr QoS of (a) $\mathrm{QoS}_{l r}=50$ and (b) $\mathrm{QoS}_{l r}=70$.

When the detector does not track the optimal threshold, thus inducing a threshold drift, the BER of the modified system is observed to be better than that of the original one for the two classes and for the two values of $P^{(l r)} / P^{(h r)}$. The threshold drift for a class- $h r$ user is $\sigma_{h r}=2$ when $P^{(l r)} / P^{(h r)}=3 / 4$ and $\sigma_{h r}=2.7$ when $P^{(l r)} / P^{(h r)}=1 / 2$. On the other hand, the threshold drift for a class-lr user is $\sigma_{l r}=3.1$ when $P^{(l r)} / P^{(h r)}=3 / 4$ and $\sigma_{l r}=3.84$ when $P^{(l r)} / P^{(h r)}=1 / 2$. Therefore, the drift increases as the power ratio deceases.

In Fig. 8, we plot the threshold line width versus the QoS guaranties for the class- $h r \mathrm{QoS}_{h r}$ when power control is used and for fixed $\mathrm{QoS}_{l r}$. Notice that the drift for class-hr users is smaller than that for class-lr users. This result was also shown theoretically in (31). When $\mathrm{QoS}_{h r}$ increases, $\sigma_{h r}$ and $\sigma_{l r}$ also increase. Observe that when we vary the multimedia pdf from $P_{h r}=P_{l r} / 2$ to $P_{h r}=P_{l r}$, the drift increases for the two classes, which confirm the result obtained in Proposition 2.

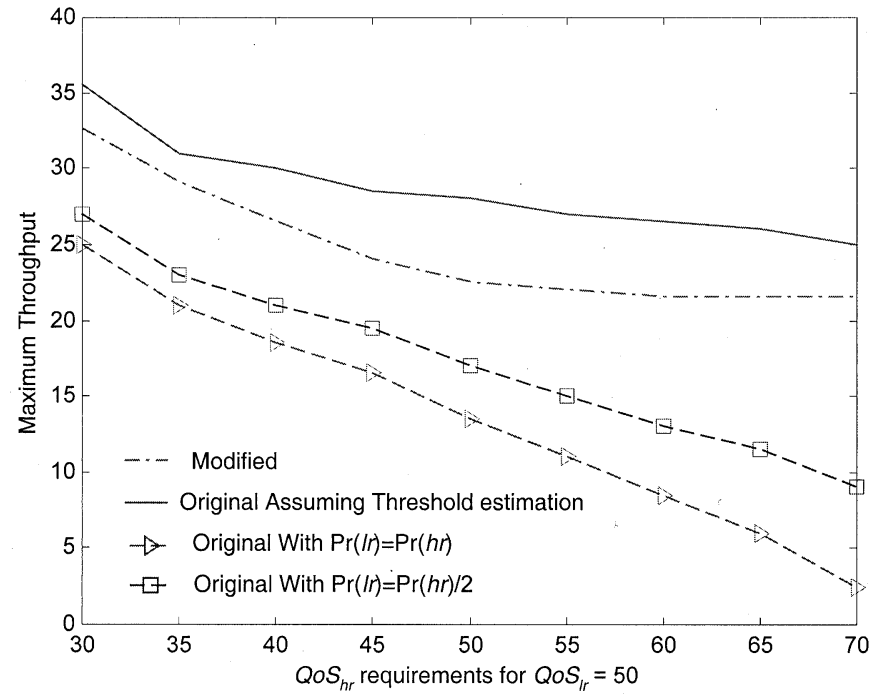

Fig. 9. Maximum throughput for the original and the modified systems versus the class-hr QoS requirement and for fixed class-lr QoS of (a) $\mathrm{QoS}_{l r}=50$ and (b) $\mathrm{QoS}_{l r}=70$.

Using the results shown in Fig. 8, Fig. 9 shows a comparison between the throughput of the original and the modified system when power control is used and when we vary the $\mathrm{QoS}_{h r}$. Simulation shows that the throughput of the original system is always higher than that of the modified system when the detector tracks the variations in the optimal threshold. On the other hand, and as predicted in previous sections, the throughput of the modified system is better when the detector does not track the variations in the optimal threshold. Moreover, when we change the multimedia pdf from $P_{l r}=P_{h r} / 2$ to $P_{l r}=P_{h r}$, the throughput of the original system decreases because the drift increases for the two classes, as shown in Fig. 8.

\section{CONCLUSION}

The average MAI fluctuations in a multirate OFFH-CDMA system has been described given a prespecified multimedia distribution and a power-control function. These fluctuations cause a drift in the optimal detection threshold, which was estimated theoretically and quantified as a variable called threshold line width. To solve this problem, a new multirate OFFH-CDMA system was proposed. The SIR and BER were evaluated for the new system using an FSV of the codes used in the original system. Results demonstrated that the new system achieves good performance without dynamic estimation of the receiver threshold. As a conclusion, this new system architecture is recommended for multirate multimedia applications.

\section{APPENDIX}

Lemma 1: Consider a discrete random variable (rv) $X$ that takes values in the interval $\left[x_{1}, x_{k}\right]$. The maximum variance of this $\mathrm{rv}$ is achieved by putting two masses of probability $1 / 2$ at the two extremes $x_{1}$ and $x_{k}$. Thus we can say that the discrete distribution that maximizes the variance of a discrete rv is the Bernoulli distribution for the set $S_{X}=\left\{x_{1}, x_{k}\right\}$ with probability masses $P_{X}=\left\{p_{1}=1 / 2, p_{k}=1 / 2\right\}$. 
Proof: Assume a discrete distribution $f(x)$ defined on the interval $x \in\left[x_{1}, x_{k}\right]$. The variance of $X$ is given by

$$
\sigma^{2}=\sum_{i=1}^{k} x_{i}^{2} f\left(x_{i}\right)-\left(\sum_{i=1}^{k} x_{i} f\left(x_{i}\right)\right)^{2} .
$$

We can always write $f(x)$ as the sum of odd and even functions $f(x)=f_{e}(x)+f_{o}(x)$. Then using the orthogonality criteria between even and odd functions, the variance in (45) can be simplified to

$$
\sigma^{2}=\sum_{i=1}^{k} x_{i}^{2} f_{e}\left(x_{i}\right)-\left(\sum_{i=1}^{k} x_{i} f_{o}\left(x_{i}\right)\right)^{2} .
$$

Due to the fact that we are seeking the maximum of $\sigma^{2}$, we must minimize the second term in (46). This is achieved by making it equal to zero. Thus we can write

$$
\sigma^{2}=\sum_{i=1}^{k} x_{i}^{2} f_{e}\left(x_{i}\right) .
$$

Equation (47) suggests that it is sufficient to obtain the maximum variance assuming $f(x)$ is even (thus symmetric about a fixed mean) in order to get the maximum over any $f(x)$. Using this result, we can restrict our analysis to the family of symmetric discrete distributions.

Suppose that $f(x)$ is a discrete distribution, which is symmetric about a fixed mean $\bar{x}=\left(x_{k}+x_{1}\right) / 2$. Thus, the variance of $x$ is given by

$$
\sigma^{2}=\sum_{i=1}^{k}\left(x_{i}-\frac{x_{k}+x_{1}}{2}\right)^{2} f\left(x_{i}\right) \quad \forall x_{i} \in\left[x_{1}, x_{k}\right]
$$

It is clear from (48) that the maximum of $\sigma^{2}$ is achieved by taking $x_{i}=x_{k}$ or $x_{i}=x_{1}$; therefore, we can write

$$
\sigma_{\max }^{2}=\frac{\left(x_{k}-x_{1}\right)^{2}}{4} .
$$

Hence, we have demonstrated that the optimal distribution that maximizes the variance is the Bernoulli distribution taken for the set $S_{X}=\left\{x_{1}, x_{k}\right\}$ with probability masses $f\left(x_{1}\right)$ and $f\left(x_{k}\right)=1-f\left(x_{1}\right)$. But knowing that $f(x)$ is an even function, this implies that $f\left(x_{k}\right)=f\left(x_{1}\right)=1 / 2$, which completes the proof of the Lemma.

\section{REFERENCES}

[1] J. G. Zhang, "Flexible optical CDMA networks using strict optical orthogonal codes for multimedia broadcasting and distribution applications," IEEE Trans. Broadcasting, vol. 45, pp. 106-115, Mar. 1999.

[2] S. Maric, O. Moreno, and C. J. Corrada, "Multimedia transmission in fiber optical LAN's using optical CDMA," IEEE J. Lightwave Technol., vol. 14, pp. 2149-2153, Oct. 1996.

[3] E. Inaty, H. M. H. Shalaby, P. Fortier, and L. A. Rusch, "Multirate optical fast frequency hopping CDMA system using power control," IEEE J. Lightwave Technol., vol. 20, pp. 166-177, Feb. 2002.

[4] H. Fathallah, L. A. Rusch, and S. Larochelle, "Passive optical fast frequency hop CDMA communication system," IEEE J. Lightwave Technol., vol. 17, pp. 397-405, Mar. 1999.

[5] A. W. Lam and A. M. Hussain, "Performance analysis of direct-detection optical CDMA communication systems with avalanche photodiode," IEEE Trans. Commun., vol. 40, pp. 810-820, Apr. 1992.

[6] T. Ohtsuki, "Performance analysis of direct-detection optical CDMA systems with optical hard-limiter using equal-weight orthogonal signaling," IEICE Trans. Commun., vol. E82-B, no. 3, pp. 512-520, Mar. 1999.

[7] E. Inaty, P. Fortier, and L. A. Rusch, "SIR performance evaluation of a multirate OFFH-CDMA system," IEEE Commun. Lett., to be published.

[8] T. Ottosson and A. Svensson, "Multi-rate performance in DS/CDMA system," Dept. of Information Theory, Charlmers Univ. of Technology, Göteborg, Sweden, Tech. Rep. 14, ISSN 0283-1260, Mar. 1995.

[9] L. D. Wronski, R. Hossain, and A. Albicki, "Extended hyperbolic congruential frequency hop code: Generation and bounds for cross- and auto-ambiguity function," IEEE Trans. Commun., vol. 44, pp. 301-305, Apr. 1996.

[10] H. L. Van Trees, Detection, Estimation and Modulation Theory, Part I. New York: Wiley, 1968.

[11] L. Cooper, Applied Nonlinear Programming for Engineers and Scientists. Englewood, NJ: Aloray, 1974.

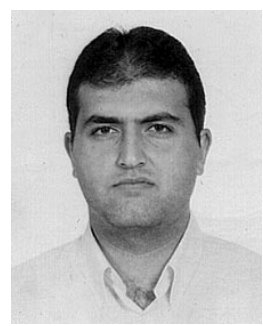

Elie Inaty (M'03) was born in Al-Koura, North Lebanon, on June 23, 1974. He received the B.S. and M.S. degrees in electrical engineering from the University of Balamand, Lebanon, in 1996 and 1998, respectively. He received the Ph.D. degree from Université Laval, QC, Canada, in 2001.

Currently, he is an Assistant Professor at the University of Balamand. His research interests include CDMA and WDM fiber-optic communications, network control, and resource management issues in optical communication networks and radio multiple

access.

Hossam M. H. Shalaby (S'83-M'83-SM'99), photograph and biography not available at the time of publication.

Paul Fortier (S'79-M'82-SM'00), photograph and biography not available at the time of publication. 